\title{
Solving Capacitated Vehicle Routing Problem with Demands as Fuzzy Random Variable
}

\author{
V. P. Singh*, Kirti Sharma and Debjani Chakraborty
}

Received: date / Accepted: date

\begin{abstract}
Capacitated vehicle routing problem (CVRP) is a classical combinatorial optimization problem in which a network of customers with specified demands is given. The objective is to find a set of routes which originates as well as terminates at the depot node. These routes are to be traversed in such a way that the demands of all the customers in the network are satisfied and the cost associated with traversal of these routes come out to be a minimum. In real-world situations, the demand of any commodity depends upon various uncontrollable factors, such as, season, delivery time, market conditions and many more. Due to these factors, the demand can always not be told in advance and a precise information about the demand is nearly impossible to achieve. Hence, the demands of the customers always experience impreciseness and randomness in real-life. The decisions made by the customers about the demands may also have some scope of hesitation as well. In order to handle such demands of customers in the network, fuzzy random variables and intuitionistic fuzzy random variables are used in this work. The work bridges the gap between the classical version of CVRP and the real-life situation and hence makes it easier for the logistic management companies to determine the routes that should be followed for minimum operational cost and maximum profit. Mathematical models corresponding to CVRP with fuzzy stochastic demands (CVRPFSD) and CVRP with Intuitionistic fuzzy stochastic demands (CVRPIFSD) have been presented. A two-stage model has been proposed to find out the solution for the same. To explain the working of the methodology defined in this work, two different example of a network with fuzzy and intuitionistic fuzzy demands have been worked out. The proposed solution approach is also tested on modified fuzzy versions of some benchmark instances.
\end{abstract}

Keywords Vehicle Routing Problem · Branch and Bound Algorithm · Discrete Fuzzy Random Variable . Fuzzy Stochastic Demands · Graded mean Integration Representation.

\section{Introduction}

CVRP [36], a very well known problem in Operational research aims at finding a set of routes beginning and ending at source node in a way such that total cost incurred comes out to be a minimum. In CVRP, we are usually given a weighted graph in which the weights on the edges represents the time(or distance or cost) needed to traverse that edge and the vertices represent customers with some demands to be fulfilled. There is a depot(source) node from where the journey of the traveling salesman [32] starts with an aim to travel each customer exactly once, fulfil their demands and then return to the depot node. In conventional CVRP, customers' demands are known precisely in advance, which makes it easier for the traveling salesman

Vishnu Pratap Singh

Department of Mathematics, Visvesvaraya National Institute of Technology Nagpur, India

E-mail: vpsingh@mth.vnit.ac.in

Kirti Sharma

Department of Mathematics, Visvesvaraya National Institute of Technology Nagpur, India E-mail: unik96kirti@gmail.com

Debjani Chakraborty

Department of Mathematics, Indian Institute of Technology Kharagpur, India

E-mail: debjani@math.iitkgp.ac.in 
to plan the routes. In daily life situations, sometimes the demand of the customers are not known clearly, whereas, sometimes, the demands may not be known well in advance.

\subsection{Literature review}

The CVRP was first proposed in 1959 by Dantzig and Ramser [10] and because of the widespread applications of the problem in logistic management, communication networks, urban solid waste collection, vehicle scheduling etc., the problem has received close attention from the optimization community since then. CVRP is an extended version of the well known Travelling Salesman problem(TSP) [9], where the objective is to determine a minimum cost Hamiltonian circuit. Thus, many approaches, may it be exact, random, approximate, heuristics based or meta-heuristics based, which are used for finding a solution for CVRP are inherited from the successful works done for finding the solution of TSP. The book edited by Toth and Vigo [36] presented an overview of various exact and heuristic methods for solving a CVRP. Exact algorithms used for solving VRP are based on Branch cut and price algorithms [16]. An interesting survey comprising of early exact methods for solving CVRP is given by Laporte and Nobert [25] in 1987.

VRP is known to be NP hard in nature; thus all the exact algorithms used for solving the problem are only applicable for small instances only and face time complexity issues for big networks. The inability of the exact approaches to solve medium and large scale vehicle routing problem as well as the difficulty in evaluating objective function in real life complex problems are two main reasons why heuristic and metaheuristics are needed for solving such problems. One of the very early works in heuristics was presented by Clark and Wright [7] in 1964, where a savings heuristics was designed to solve the problem. A heuristic method based on nearest neighbour algorithm was defined in [30]. Nearest neighbour algorithm is a greedy algorithm in which the nearest vertex is traversed first when given a set of vertices to be traversed. A tabu search algorithm was presented by Ubeda et. al in [37] for solving a green CVRP. In tabu search method, a local heuristic method is used to explore the solution space beyond optimality. Meta-heuristic methods based on genetic algorithm was presented in [28]. Genetic algorithm mimics the natural evolution process as defined in theory of evolution by Charles Darwin. Meta-heuristic methods based on ant colony optimization and particle swarm optimization were presented in [11] and [14] respectively. Such method imitates the behaviour of ants and swarms and a local optimal solution is obtained. A hybrid genetic algorithm - bacteria foraging optimization algorithm was defined in [2]. A hybrid particle swarm optimization and simulated annealing method was presented in [14]. Christofides heuristic method was extended by Frieze et. al in [15] to solve a k-person TSP. A modified fuzzy C-means clustering approach was presented in [33] for solving CVRP. The approach is based on cluster first route second method. In [33], the first stage of problem solving corresponds to the formation of clusters and in second phase, each cluster is solved independently as a TSP.

Recent years have witnessed various variants of CVRP based on the uncertainty and variability of different attributes of the problem. In the classical version of the problem, all the information is available well in advance and such a VRP is known as a Dynamic VRP. If one or more parameters of the problem are not known well in advance, then such a problem is known as Stochastic VRP(SVRP). The early works in SVRP were presented in [17], [12] and [25]. The stochastic nature of the problem is generally found in two parameters, namely the demands of the customers and the travel times. Cordeau et. al [8] studied the most basic and most studied version of SVRP, i.e., CVRP with stochastic demands (CVRPSD). In CVRPSD, the customer demands are not known well in advance and are only revealed when a vehicle arrives at a customer location. In [3], a robust solution approach was presented for solving dynamic and stochastic vehicle routing problem. A two stage stochastic program model with recourse is presented where the objective of first stage is to minimize the a-priori routing plan and the second stage dealt with minimizing the recourse cost. A local search based algorithm with the objective of minimizing the total number of vehicles used and total distance visited was developed in [22] for solving CVRPSD. In [26], a case study of real-life problem of municipal waste collection in the city of Nis is considered and a solution approach based on heuristic and meta-heuristic methods was presented for solving CVRPSD. In [39], a hybrid algorithm called DSMO-GA which combines genetic algorithm(GA) and discrete spider monkey optimization (DSMO) algorithm was proposed for solving CVRPSD.

When the customer demands are not known precisely but are given as uncertain quantities, i.e., as fuzzy numbers, then such a VRP is known as VRP with fuzzy demands. In [35], CVRP with fuzzy demands given by interval type-2 triangular fuzzy numbers was presented. They used a modified Clarke and Wright algorithm to solve the problem. In [38], CVRP with fuzzy demands was handled by using fuzzy 
multi-criteria modelling approach. In [24], hybrid particle swarm optimization with a genetic algorithm for solving CVRP with fuzzy demands is proposed. In [27], a location arc routing problem was solved by using a mixed integer programming model. In [27], the demand of each edge belongs to a bounded uncertainty set. The proposed model determines a subset of potential depots to be opened along with their allocated routes. In [13], a bi-objective green vehicle routing problem with heterogeneous fleet is proposed. Along with the minimization of distance, the green house gases emitted by the vehicles during the operation is also minimized. Multi-criteria optimization and VIKOR method are used to determine the best solution from the Pareto front.

In certain situations, the cost matrix stores the time taken to traverse edges in the network. In many practical problems, the travel time between two locations in a routing problem cannot be told precisely because of traffic congestion or road conditions. Such situations can be handled by using fuzzy numbers for representing travel times. A fuzzy approach for solving VRP with fuzzy travel times was presented in [4]. There also exist situations when the travel times can not be told well in advance. Such a version of CVRP with stochastic travel times was presented in [29]. In [29], a non-dominated sorting genetic algorithm (NSGA) together with a variable neighbourhood search (VNS) heuristic was proposed. The demands of the customers were assumed to be deterministic. In addition to stochastic travel time, a soft time window is also associated with every customer and there exist a penalty for serving the customer outside their specified time window. In [41] a hybrid ant colony optimization and genetic algorithm is used to solve VRPTW(VRP with time windows) where the demands of the customers, as well as travel time between every pair of customers, is imprecise. In [19], a particular case of VRP where triangular fuzzy numbers are used to represent the fuzzy travel times and the deliveries are split into bags was modelled. In [18], an algorithm based on Clarke and Wright savings heuristic where three criterion of the network namely the distance of various edges, time required to travel these edges and road quality are considered as fuzzy. In [23], fuzzy and evolutionary algorithms were used for planning the routes of tankers when the demand of fuel at various nodes in the network is presented by using Triangular fuzzy numbers.

\subsection{Novelty and Contribution}

Higher cost of precise information retrieval and random nature of demands of customers give rise to CVRPFSD. In CVRPFSD, the demands of the customers are neither presented precisely and are also not given well in advance. The decisions about the demands also face hesitation because of fluctuating demand-supply chain network. This work considers such fuzzy stochastic nature of customers' demands and such nature of customer demands is handled by using fuzzy random variables. In this work, the basic version of CVRPSD has been extended and the current version deals with CVRPFSD and CVRPIFSD. In this paper, the edge weights denote the travel cost. The customers' demands are only revealed upon the arrival of the vehicle and even then the demands of the customers are not told precisely. Various uncontrollable factors like delivery hours, market conditions, demand-supply chain fluctuations contribute to this imprecise and random nature of the customers' demands. In this work, two cases have been considered. The first case corresponds to the situation when the demands of customers are represented by using fuzzy random variables whereas in the second case, the demands of the customers are represented by using intuitionistic fuzzy random variables. Various methods have been developed in literature for solving CVRP with fuzzy demands and CVRPSD separately but a combination of randomness and impreciseness have never been dealt in the literature. To the best of author's knowledge, such an amalgamation of randomness and impreciseness will bridge the gap between conventional and real-life problems.

In this work, CVRPFSD(CVRPIFSD) has been modelled as a two-level stochastic process. While solving the problem, the first task is to design a minimum cost path which traverse each node exactly once. Here, we used Branch and Bound algorithm for finding a minimum cost Hamiltonian circuit. The branch and bound algorithm can only be used for symmetric networks of small sizes and this comes up as a major limitation of this work. After designing, the execution of these routes takes place. Since the demands of the customers are not known in advance and are imprecise in nature, it may happen that upon reaching a customer, the salesman will realize his/her inability to serve the customer. This is the case when route failure occurs and in such cases, the salesman is required to return to the depot node, replenish their vehicle and then re-continue the planned route from the point of recent failure. In this work, the customer is served only in one visit of one vehicle, i.e., un-split delivery service policy is followed. The recourse policy followed in this work is reactive in nature, i.e., a vehicle will only return to depot when a route failure will occur. The results obtained by this method can only be used when the demands of the customers are represented 
by discrete fuzzy (intuitionistic fuzzy) random variables, where the demand of the customers and their respective probability are given by triangular (triangular intuitionistic) fuzzy numbers. The results of this work can be extended for scheduling problems faced by service agents since the service time windows are always stochastic and imprecise. The model can also be further extended to include the randomness and impreciseness of the network and such networks can be represented by using neutrosophic graph theory [21]. The work can also be extended when the demands of the customers present in the network are expressed by using linguistic environment using dual-connection numbers [20]. The use of dual connection numbers for representing demands will help the customers to formulate the membership degree under certain and uncertain situations and thus help the customers in making feasible and rational judgements.

\subsection{Applications of CVRPFSD and CVRPIFSD}

Real world applications of the CVRPFSD and CVRPIFSD include the distribution of perishable items from stock-holders to retailers, who further sell it to the consumers. For this work, the retailers play the role of customers and the stock-holders play the role of travelling salesman. Since perishable items decay after a period of time which usually depend upon the uncontrollable weather conditions. So, the demands of perishable items are always random in nature. Factors like the fluctuations of market supply and demand of any perishable item make it difficult for the retailers to precisely determine the demand. In such case, the demands are told randomly and imprecisely. Such real-life situations give rise to CVRPFSD. Psychologically, the experience of retailers also make them hesitant because an unreasonable stock of such items would only either lead to decay and hence the loss (if more stock is bought), in terms of money or it will lead to unsatisfied consumers (if less stock is bought), which will cost their market reputation. So, the decision of the retailer has some scope of hesitancy in addition to the randomness and impreciseness while entailing their demand. Such real-life situations give rise to CVRPIFSD. Another applications includes distribution of cash to different automatic teller machines (ATMs) in the city. Other examples include the delivery of essential commodity (milk, oil) where daily customer consumption is random in nature but can be predicted with the use of discrete random variable.

This paper is structured as follows: Section 2 deals with the basic concepts and definitions of fuzzy set theory and intuitionistic fuzzy set theory. Branch and Bound algorithm for solving TSP is also presented. In section 3, two mathematical models for solving CVRP in an imprecise and random environment when the demands of the customers are represented by using triangular fuzzy numbers and triangular intuitionistic fuzzy number have been presented. Section 4 deals with a procedure based on branch and bound algorithm to solve CVRPFSD and CVRPIFSD. This section comprises of a two-level algorithm and a two-level flowchart which presents the working of the two-level stochastic process defined in this paper. In section 5, two numerical examples with customers having fuzzy stochastic demands and intuitionistic fuzzy stochastic demands have been presented. The section 5 also comprises of the comparison of the results obtained by the other methods and the method proposed in this work and thus explains the supremacy of the method proposed. The proposed algorithm is also tested on the modified version of some benchmark instances. Section 6 comprises of the concluding remarks.

\section{Preliminaries: Concepts and Definitions}

Definition 1 Fuzzy Number : A fuzzy set [40] $\tilde{A}$ on $\mathrm{R}$ is said to be a fuzzy number, if the following three properties are satisfied:

1. Fuzzy set $\tilde{A}$ must be normal, i.e. $\exists x$ such that $\sup \mu_{\tilde{A}}(x)=1$.

2. The support of fuzzy set, i.e. set of all the elements with non zero degree of membership, must be bounded.

3. $\alpha$ level set i.e. set of all the elements with membership degree greater than $\alpha$, must be a closed interval for $\alpha \in[0,1]$. 
Definition 2 Triangular Fuzzy Number : A generalized triangular fuzzy number is written as $\tilde{A}=(a, b, c)$ and the membership function of triangular fuzzy number $(a, b, c)$ is given by eq. (1).

$$
\mu_{\tilde{A}}(x)= \begin{cases}0 & \text { if } x \leq a \text { and } x>c \\ \frac{x-a}{b-a} & \text { if } a<x \leq b \\ \frac{c-x}{c-b} & \text { if } b<x \leq c\end{cases}
$$

Definition 3 Symmetric Triangular Fuzzy number : A triangular fuzzy number is said to be symmetric if its left and right spreads are equal. If $\tilde{A}=(a, b, c)$ is a triangular fuzzy number, then it is said to be symmetric if and only if $(b-a)=(c-b)$.

Example $1(2,4,6)$ is a symmetric triangular fuzzy number. The graph of the membership function of a symmetric triangular fuzzy number is given by Figure 1:

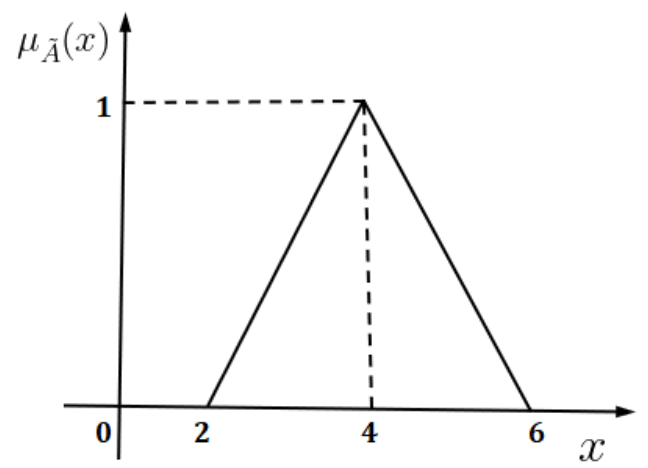

Fig. 1: A Symmetric Triangular Fuzzy Number

Definition 4 Arithmetic operations on Symmetric Triangular Fuzzy Number : Let $\tilde{A}=\left(a_{1}, b_{1}, c_{1}\right)$ and $\tilde{B}=\left(a_{2}, b_{2}, c_{2}\right)$ are two symmetric triangular fuzzy numbers, then

1. Addition:

$$
\left(a_{1}, b_{1}, c_{1}\right) \oplus\left(a_{2}, b_{2}, c_{2}\right)=\left(a_{1}+a_{2}, b_{1}+b_{2}, c_{1}+c_{2}\right)
$$

\section{Symmetric Image:}

$$
-\left(a_{1}, b_{1}, c_{1}\right)=\left(-c_{1},-b_{1},-a_{1}\right)
$$

3. Subtraction:

$$
\left(a_{1}, b_{1}, c_{1}\right) \ominus\left(a_{2}, b_{2}, c_{2}\right)=\left(a_{1}-c_{2}, b_{1}-b_{2}, c_{1}-a_{2}\right)
$$

4. Multiplication:

$$
\left(a_{1}, b_{1}, c_{1}\right) \odot\left(a_{2}, b_{2}, c_{2}\right)=\left(b_{1} a_{2}+b_{2} a_{1}-b_{1} b_{2}, b_{1} b_{2}, b_{1} c_{2}+b_{2} c_{1}-b_{1} b_{2}\right)
$$

for $\tilde{A}$ and $\tilde{B}$ positive;

Definition 5 Triangular Intuitionistic Fuzzy Number (TIFN) : A Triangular Intuitionistic fuzzy number (TIFN) $[1] \tilde{A}^{I}$ is an intuitionistic fuzzy number with the membership function and non-membership function given by eq. (2) and eq. (3) respectively.

$$
\mu_{\tilde{A}^{I}}(x)=\left\{\begin{array}{ll}
\frac{x-a}{b-a} & \text { if } a<x<b \\
\frac{c-x}{c-b} & \text { if } x=b \\
0 & \text { if } b<x \leq c
\end{array} \quad(2) \quad \vartheta_{\tilde{A}^{I}}(x)= \begin{cases}\frac{b-x}{b-a^{\prime}} & \text { if } a^{\prime}<x<b \\
0 & \text { if } x=b \\
\frac{x-c^{\prime}}{c^{\prime}-b} & \text { if } b<x \leq c^{\prime} \\
1 & \text { otherwise }\end{cases}\right.
$$


where $a^{\prime} \leq a \leq b \leq c \leq c^{\prime}$. This TIFN is denoted by $\left(a, b, c ; a^{\prime}, b, c^{\prime}\right)$. Figure 2 represents the membership and non-membership functions of TIFN defined above.

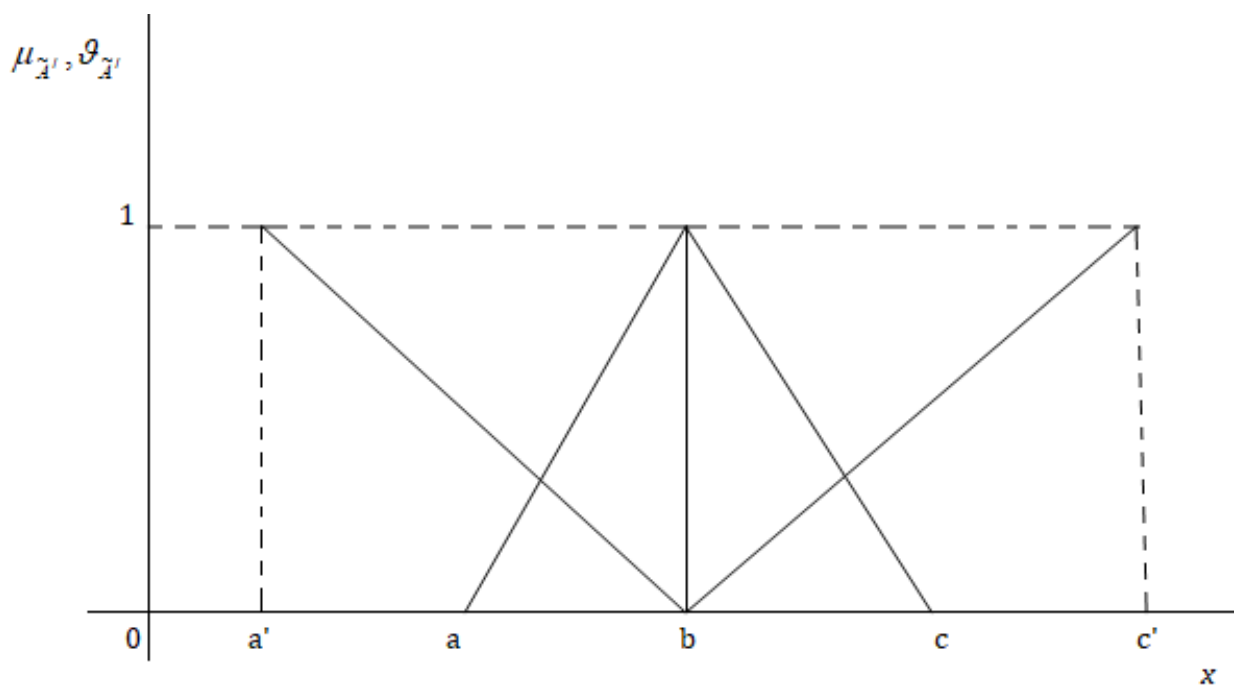

Fig. 2: A Triangular Intuitionistic Fuzzy Number

Definition 6 Arithmetic Operations on TIFNs [1]: Let $\tilde{A}^{I}=\left(a, b, c ; a^{\prime}, b, c^{\prime}\right)$ and $\tilde{B}^{I}=\left(p, q, r ; p^{\prime}, q, r^{\prime}\right)$ be two TIFNs, then

\section{Addition:}

$$
\tilde{A}^{I} \oplus \tilde{B^{I}}=\left(a+p, b+q, c+r ; a^{\prime}+p^{\prime}, b+q, c^{\prime}+r^{\prime}\right)
$$

\section{Subtraction:}

$$
\tilde{A}^{I} \ominus \tilde{B^{I}}=\left(a-r, b-q, c-p ; a^{\prime}-r^{\prime}, b-q, c^{\prime}-p^{\prime}\right)
$$

3. Multiplication:

$$
\tilde{A}^{I} \odot \tilde{B}^{I}=\left(l_{1}, l_{2}, l_{3} ; l_{1}^{\prime}, l_{2}, l_{3}^{\prime}\right)
$$

where

$$
\begin{array}{lr}
l_{1}=\min \{a p, a r, c p, c r\} & l_{3}=\max \{a p, a r, c p, c r\} \\
l_{1}^{\prime}=\min \left\{a^{\prime} p^{\prime}, a^{\prime} r^{\prime}, c^{\prime} p^{\prime}, c^{\prime} r^{\prime}\right\} & l_{3}^{\prime}=\max \left\{a^{\prime} p^{\prime}, a^{\prime} r^{\prime}, c^{\prime} p^{\prime}, c^{\prime} r^{\prime}\right\} \\
l_{2}=b q &
\end{array}
$$

\section{Scalar Multiplication:}

$$
k \tilde{A^{I}}= \begin{cases}\left(k a, k b, k c ; k a^{\prime}, k b, k c^{\prime}\right) & \text { if } k \geq 0 \\ \left(k c, k b, k a ; k c^{\prime}, k b, k a^{\prime}\right) & \text { if } k<0\end{cases}
$$

\section{Division:}

$$
\tilde{A^{I}} / \tilde{B^{I}}=\left\{\frac{a}{r}, \frac{b}{q}, \frac{c}{p} ; \frac{a^{\prime}}{r^{\prime}}, \frac{b}{q}, \frac{c^{\prime}}{p^{\prime}}\right\}
$$

where $a \geq 0, p>0$

Definition 7 Graded Mean Integration Representation: [5] Let $L^{-1}$ and $R^{-1}$ are the inverse functions of $L$ (left) and $R$ (right) respectively, then the graded mean integration representation of a generalized triangular fuzzy number $\tilde{A}$ is given by eq. (4).

$$
G(\tilde{A})=\frac{\int_{0}^{1} h\left(\frac{L^{-1}(h)+R^{-1}(h)}{2}\right) d h}{\int_{0}^{1} h d h}
$$

Thus, the graded mean integration representation [6] of a triangular fuzzy number $\tilde{A}=(a, b, c)$ is given by eq. (5).

$$
G(\tilde{A})=\frac{a+4 b+c}{6}
$$


Definition 8 Accuracy Function: Let $\tilde{A}^{I}=\left(a, b, c ; a^{\prime}, b, c^{\prime}\right)$ be a triangular intuitionistic fuzzy number. The score function for membership function and non-membership function is denoted by $S\left(\mu_{\tilde{A}^{I}}\right)$ and $S\left(\vartheta_{\tilde{A}^{I}}\right)$ respectively where

$$
S\left(\mu_{\tilde{A}^{I}}\right)=\frac{a+2 b+c}{4} \quad \text { and } \quad S\left(\vartheta_{\tilde{A}^{I}}\right)=\frac{a^{\prime}+2 b+c^{\prime}}{4} .
$$

The accuracy function [34] of $\tilde{A}^{I}$ is denoted by $f\left(\tilde{A}^{I}\right)$ and is defined by eq. (6).

$$
f\left(\tilde{A}^{I}\right)=\frac{(a+2 b+c)+\left(a^{\prime}+2 b+c^{\prime}\right)}{8}
$$

Definition 9 Fuzzy Random Variable: A fuzzy random variable [31] is a random variable whose value is not real, but a fuzzy number.

Definition 10 Expectation of Discrete Fuzzy Random Variable: If $\tilde{X}$ is a discrete fuzzy random variable, in such a way that $P\left(\tilde{X}=\tilde{x}_{i}\right)=\tilde{p_{i}}, \mathrm{i}=1,2,3, \cdots$, then the fuzzy expectation is given by

$$
E \tilde{X}=\sum_{i=1}^{n} \tilde{x_{i}} \odot \tilde{p_{i}} .
$$

\subsection{Branch and Bound Algorithm [9]}

The Branch and Bound algorithm is used to find exact solution for a wide variety of combinatorial optimization problems like TSP, knapsack problem, job scheduling problem and many more. The algorithm works by systematically enumerating all the candidates solutions and then discarding some useless candidates by using the estimated bound on the quantity being optimized. In Branch and Bound algorithm, a tree search strategy is used to enumerate all possible solutions of a given optimization problem and only those branches of tree are explored which satisfies a bound. Figure 3 shows a branch and bound tree search strategy corresponding to a network with 4 nodes. To avoid unnecessary exploration of entire tree, the algorithm calculates a value (known as bound) corresponding to each node. The bound of any node represent the cost of best possible solution that can be obtained if a path through that node is traversed. If a better solution cannot belong to the sub tree rooted at a considered node, then the sub tree need not be further explored. Otherwise, the process of exploration continues. For minimization problem, a lower bound corresponding to every node is calculated and the branches with lesser lower bounds are explored for better solution. For maximization problem, an upper bound corresponding to every node is calculated and the branches with higher upper bounds are explored first since that will provide better solution.

\section{Mathematical Model}

In this section, mathematical models for solving a CVRP with random and imprecise demands have been formulated. The mathematical models depicted in subsection 3.1 and subsection 3.2 represent the situation when the demands of the customers are given by discrete fuzzy random variables and impreciseness of customer demands is handled by using triangular fuzzy numbers and TIFNs respectively.

In this work, some assumptions regarding the structure of the network, demands of the customers in the network, the recourse policy and the service policy used have been made. The structure of the network is assumed to be symmetric, i.e. $c_{i j}=c_{j i}$, which means that the cost of traversal from node $i$ to node $j$ is same as the cost of traversal from node $j$ to node $i$. The demands of the customers are assumed to be independent and positive. The demand of every individual customer is assumed to be less than the carrying capacity of the vehicle used to maintain the feasibility of the network. In this work, the factors like randomness and impreciseness have been used to represent the demands of the customer. The random(stochastic) nature of the customer demands means that the demands of the customers are only known when the vehicle arrives and this stochastic nature of demands lead to the failure of the route. The failure of route means that when a salesman arrives at a particular customer, they realize that they don't have enough capacity of goods to serve the customer. In this work, it has been assumed that the route failure may occur any number of times. Upon the failure of the route, the salesman is supposed to return to the depot, i.e., a reactive recourse policy is used. After returning to the depot, the vehicles are supposed to replenish themselves and continue the service again. In this work, it has been assumed that each customer is visited exactly once and is served in that visit only, i.e., the delivery policy used is un-split in nature. The journey of the travelling salesman is 


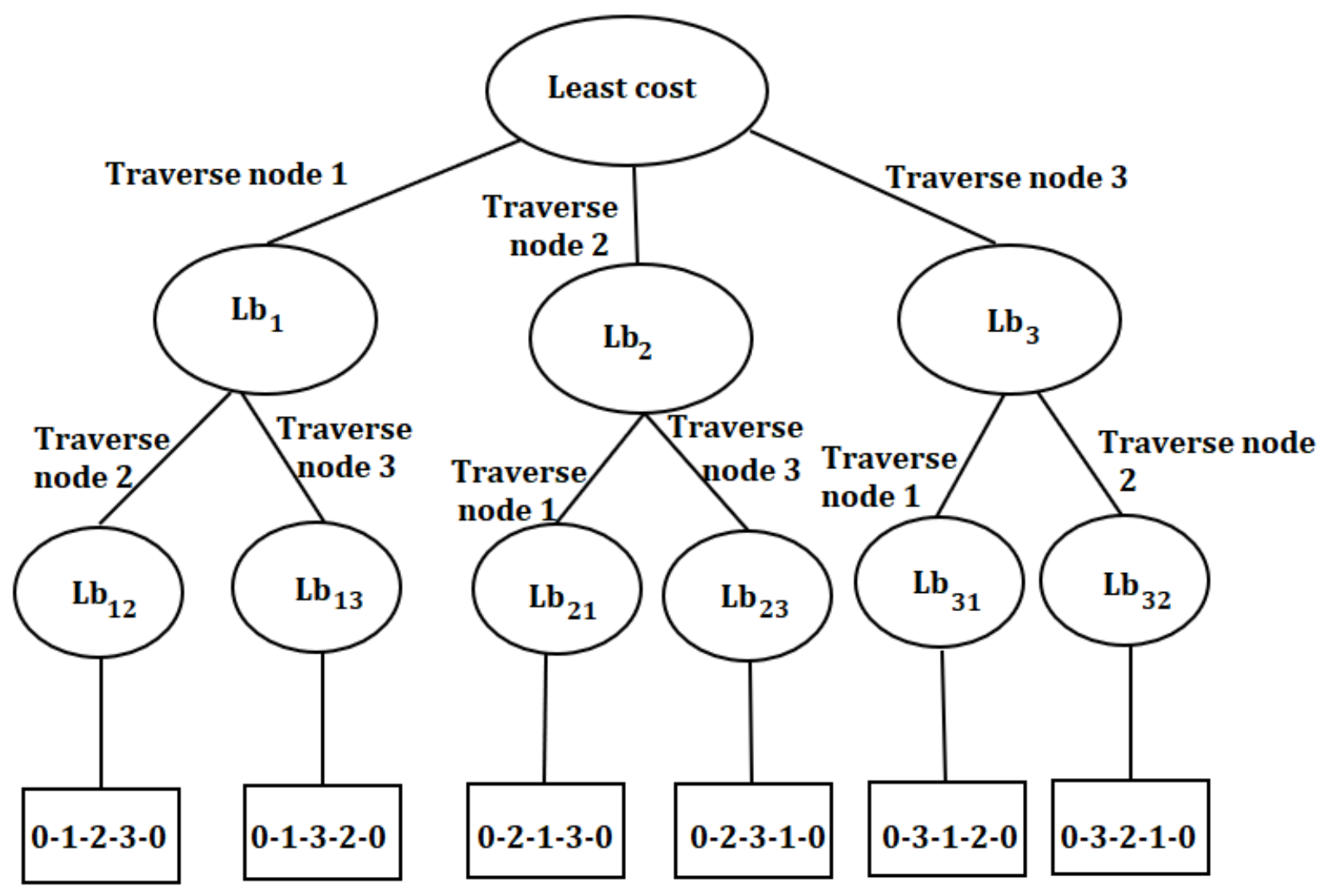

Fig. 3: A tree based search strategy for TSP in a network with 4 nodes

always assumed to originate and terminate at depot node only. The fleet used for serving the customers is assumed to be homogeneous, i.e. all the vehicles have the same carrying capacity and they all operate at the identical cost.

We will now formulate the mathematical model for CVRP with Fuzzy Stochastic Demands. Let $G=$ $\left(N=C \cup\left\{s, s^{\prime}\right\}, A\right)$ be a graph. The notation $C$ is used for the set of customers and $s$ and $s^{\prime}$ represents the source node and its copy, respectively. $c_{i j}$ represents the cost of travelling from node $i$ to node $j$. Let $\widetilde{D_{i}}$ and $\widetilde{D_{i}^{I}}$ be the fuzzy random variable and intuitionistic fuzzy random variable representing the fuzzy and intuitionistic fuzzy demands of the customer at node $i$ respectively. Demand of source node is assumed to be 0 units.

A route is defined as a path of the form $P=\left(p_{1}, p_{2}, \cdots, p_{|P|}\right)$ where $p_{1}=s$ and $p_{|P|}=s^{\prime}$ with $p_{h} \in C$ for $h \in\{2,3,4, \cdots|k|-1\}$. Let $\widetilde{T D}\left(\widetilde{\mu_{p_{h}}}, \widetilde{\sigma_{p_{h}}^{2}}\right)=\sum_{i=1}^{h} \widetilde{D_{p_{i}}}$ and $\widetilde{T D^{I}}\left(\widetilde{\mu_{p_{h}}^{I}}, \widetilde{\sigma^{2}{ }_{p_{h}}}\right)=\sum_{i=1}^{h} \widetilde{D_{p_{i}}^{I}}$ denotes the total actual fuzzy cumulative demand and the total actual intuitionistic fuzzy cumulative demand at the customer $p_{h}$ for $h \in\{2,3,4, \cdots|P|-1\}$.

Given a route $P=\left(p_{1}, p_{2}, \cdots, p_{|P|}\right)$, let $\widetilde{E F C}\left(\widetilde{\mu_{p_{h}}}, \widetilde{\sigma_{p_{h}}^{2}}\right)$ and $\widetilde{E F C^{I}}\left(\widetilde{\mu^{I}}{ }_{p_{h}}, \widetilde{\sigma^{2}{ }^{I}}{ }_{p_{h}}\right)$ denotes the expected fuzzy and expected intuitionistic fuzzy failure cost at customer $p_{h}$ respectively. It can be written as,

$$
\widetilde{E F C}\left(\widetilde{\mu}_{p_{h}}, \widetilde{\sigma^{2} p_{h}}\right)=2 c_{0 p_{h}}\left(\sum_{u=1}^{\infty}\left(P\left(G\left(\widetilde{T D}\left(\widetilde{\mu}_{p_{h-1}}, \widetilde{\sigma^{2} p_{h-1}}\right)\right) \leq u Q\right)-P\left(G\left(\widetilde{T D}\left(\widetilde{\mu}_{p_{h}}, \widetilde{\sigma^{2}} p_{p_{h}}\right)\right) \leq u Q\right)\right)\right)
$$


$\widetilde{E F C^{I}}\left(\widetilde{\mu^{I}}{ }_{p_{h}}, \widetilde{\sigma^{2 I}}{ }_{p_{h}}\right)=2 c_{0 p_{h}}\left(\sum_{u=1}^{\infty}\left(P\left(f\left(\widetilde{T D^{I}}\left(\widetilde{\mu^{I}}{ }_{p_{h-1}}, \widetilde{\sigma^{2}}{ }_{p_{h-1}}\right)\right) \leq u Q\right)-P\left(f\left(\widetilde{T D}\left(\widetilde{\mu^{I}}{ }_{p_{h}}, \widetilde{\sigma^{2}{ }_{p_{h}}}\right)\right) \leq u Q\right)\right)\right)$

$P\left(G\left(\widetilde{T D}\left(\widetilde{\mu}_{p_{h-1}}, \widetilde{\sigma^{2}} p_{p_{h-1}}\right)\right) \leq u Q\right)-P\left(G\left(\widetilde{T D}\left(\widetilde{\mu}_{p_{h}}, \widetilde{\sigma^{2}} p_{h}\right)\right) \leq u Q\right)$ therefore indicates the fuzzy probability of having the $u^{\text {th }}$ failure at $p_{h}$ customer when the demands are given by discrete fuzzy random variables with the condition that failure has yet not occurred on any other node which has been previously visited on the route. $P\left(f\left(\widetilde{T D^{I}}\left(\widetilde{\mu^{I}}{ }_{p_{h-1}}, \widetilde{\sigma^{2}{ }_{p_{h-1}}}\right)\right) \leq u Q\right)-P\left(f\left(\widetilde{T D}\left(\widetilde{\mu^{I}}{ }_{p_{h}}, \widetilde{\sigma^{2 I}} p_{h}\right)\right) \leq u Q\right)$ thus indicates the intuitionistic fuzzy probability of having the $u^{\text {th }}$ failure at $p_{h}$ customer under the same conditions as explained for the case when demands are given by fuzzy random variables.

3.1 Mathematical model for CVRPFSD

Minimize

$$
\left.\tilde{c}_{p}=\sum_{h=1}^{|P|-1}\left(c_{p_{h} p_{h+1}}+\widetilde{E F C}_{p_{h+1}}\left(\widetilde{\mu}_{p_{h+1}}, \widetilde{\sigma^{2}} p_{h+1}\right)\right)\right)
$$

subject to

$$
\begin{aligned}
\sum_{p \in R} \alpha_{i p} \lambda_{p} & =1, \quad \forall i \in N \\
\sum_{p \in R} \lambda_{p} & \geq \vartheta_{F}(n) \\
\lambda_{p} & \in\{0,1\} \quad \forall p \in R
\end{aligned}
$$

3.2 Mathematical model for CVRPIFSD

Minimize

$$
\left.\tilde{c}_{p}=\sum_{h=1}^{|P|-1}\left(c_{p_{h} p_{h+1}}+{\widetilde{E F C^{I}}}_{p_{h+1}}\left(\widetilde{\mu^{I}} p_{p_{h+1}}, \widetilde{\sigma^{2 I}}{ }_{p_{h+1}}\right)\right)\right)
$$

subject to

$$
\begin{aligned}
\sum_{p \in R} \alpha_{i p} \lambda_{p} & =1, \quad \forall i \in N \\
\sum_{p \in R} \lambda_{p} & \geq \vartheta_{I F}(n) \\
\lambda_{p} & \in\{0,1\} \quad \forall p \in R
\end{aligned}
$$

In the mathematical models proposed above, $\alpha_{i p}$ and $\lambda_{p}$ are binary decision variables. The value of $\lambda_{p}$ is 1 if route $p$ is chosen and 0 otherwise. $\alpha_{i p}$ is also a binary decision variable which takes the value 1 if the node $i$ is traversed while traversing the route $p$. R is the set of all feasible routes originating and terminating at the source node. The term $\vartheta_{F}(n)$ and $\vartheta_{I F}(n)$ are lower bounds on the number of vehicles required when the service is performed by a fleet of vehicles or the number of times the vehicle may be required to return to source node because of failure in fulfilling the fuzzy and intuitionistic fuzzy demands of the customers respectively, if only one vehicle is used. These can be easily computed by the formulas given in eq. (17) and eq. (18) respectively.

$$
\begin{gathered}
\vartheta_{F}(n)=\left\lceil\frac{G\left(\sum_{i \in N} E\left[\tilde{D}_{i}\right]\right)}{Q}\right\rceil \\
\vartheta_{I F}(n)=\left\lceil\frac{f\left(\sum_{i \in N} E\left[\tilde{D}_{i}{ }^{I}\right]\right)}{Q}\right\rceil
\end{gathered}
$$


where $Q$ is the capacity of the vehicle.

The objectives of minimization of operation cost are represented by eq. (9) and eq. (13) for CVRPFSD and CVRPIFSD respectively. The condition of serving each customer exactly once are represented by eq. (10) and eq. (14). The use of minimum number of vehicles needed for successful execution is governed by eq. (11) and eq. (15) for CVRPFSD and CVRPIFSD respectively. The constraint fro route selection for CVRPFSD and CVRPIFSD are represented by eq. (12) and eq.(16) respectively.

Table 1: Description of symbols used in Mathematical model

\begin{tabular}{|c|c|}
\hline Symbol & Description of the symbol \\
\hline $\mathrm{C}$ & Set of customers in the network. \\
\hline$c_{i j}$ & Cost of traversal of edge $i j$ \\
\hline$P=\left(p_{1}, p_{2}, \cdots, p_{|P|}\right)$ & $\begin{array}{l}\text { a route starting and ending at } p_{1} \\
\text { and } p_{|P|} \text { respectively. }\end{array}$ \\
\hline$\widetilde{D_{i}}$ & $\begin{array}{l}\text { A fuzzy random variable representing } \\
\text { the demands of customer at node } p_{i}\end{array}$ \\
\hline$\widetilde{D_{i}^{I}}$ & $\begin{array}{l}\text { An intuitionistic fuzzy random variable } \\
\text { representing the demands of the custo- } \\
\text { mer at node } p_{i}\end{array}$ \\
\hline$P(E)$ & Probability of an event $E$. \\
\hline$\widetilde{T D}\left(\tilde{\mu}_{p_{h}}, \tilde{\sigma}_{p_{h}}^{2}\right)$ & $\begin{array}{l}\text { Fuzzy cumulative demand at customer } \\
p_{h} \text { in the route } p\end{array}$ \\
\hline$\widetilde{T D^{I}}\left(\widetilde{\mu^{I}}{ }_{p_{h}}, \widetilde{\sigma^{2 I}}{ }_{p_{h}}\right)$ & $\begin{array}{l}\text { Intuitionistic Fuzzy cumulative demand } \\
\text { at customer } p_{h} \text { in the route } p\end{array}$ \\
\hline$Q$ & capacity of the vehicle \\
\hline$G(\tilde{A})$ & GMIR representation of $\tilde{A}$ \\
\hline$f\left(\tilde{A}^{I}\right)$ & Score function of TIFN $\tilde{A}^{I}$ \\
\hline$\widetilde{E F C^{I}}\left(\widetilde{\mu^{I}}{ }_{p_{h}}, \widetilde{\sigma^{2}{ }^{I}}{ }_{p_{h}}\right)$ & $\begin{array}{l}\text { Fuzzy effective failure cost at customer } \\
p_{h} \text { of the route } P\end{array}$ \\
\hline$\lambda_{p}$ & $\begin{array}{l}\text { a binary decision variable whose value is } \\
1 \text { when route } P \text { is traversed and } 0 \text { otherwise }\end{array}$ \\
\hline$\alpha_{i p}$ & $\begin{array}{l}\text { a binary decision variable whose entry is } \\
1 \text { when node } i \text { is traversed in route } P \\
\text { and } 1 \text { when node } i \text { is traversed in route } P \text {. }\end{array}$ \\
\hline$R$ & set of all the routes \\
\hline$E[\tilde{X}]$ & Expected value of a fuzzy random variable $X$ \\
\hline$\vartheta_{F}(n)\left(\vartheta_{I F}(n)\right)$ & $\begin{array}{l}\text { Lower bound on the number of vehicles when } \\
\text { demands are given by Fuzzy (intuitionistic } \\
\text { fuzzy) random variable }\end{array}$ \\
\hline
\end{tabular}

\section{Methodology}

In this work, a two-level stochastic process has been defined for solving CVRPFSD and CVRPIFSD. The first level corresponds to the determination of an a priori route and the cost associated with that route. Various algorithms used for solving a TSP can be used for this purpose. In this work, we have used Branch and Bound algorithm for this purpose because the method ensures an optimal solution. The second level corresponds to the execution of the a priori route found in the first level and serving the customers. While executing the a priori route, a route failure may occur and in the second stage, an effective fuzzy failure cost corresponding to every customer in the network is calculated. The sum of deterministic cost obtained in stage 1 and effective failure cost obtained in stage 2 gives the cost of operation.

\subsection{Algorithm}

The stage 1 of methodology is represented by Algorithm 1. The stage 2 of methodology for fuzzy and intuitionistic fuzzy demands are represented by Algorithm 2 and Algorithm 3 respectively. 


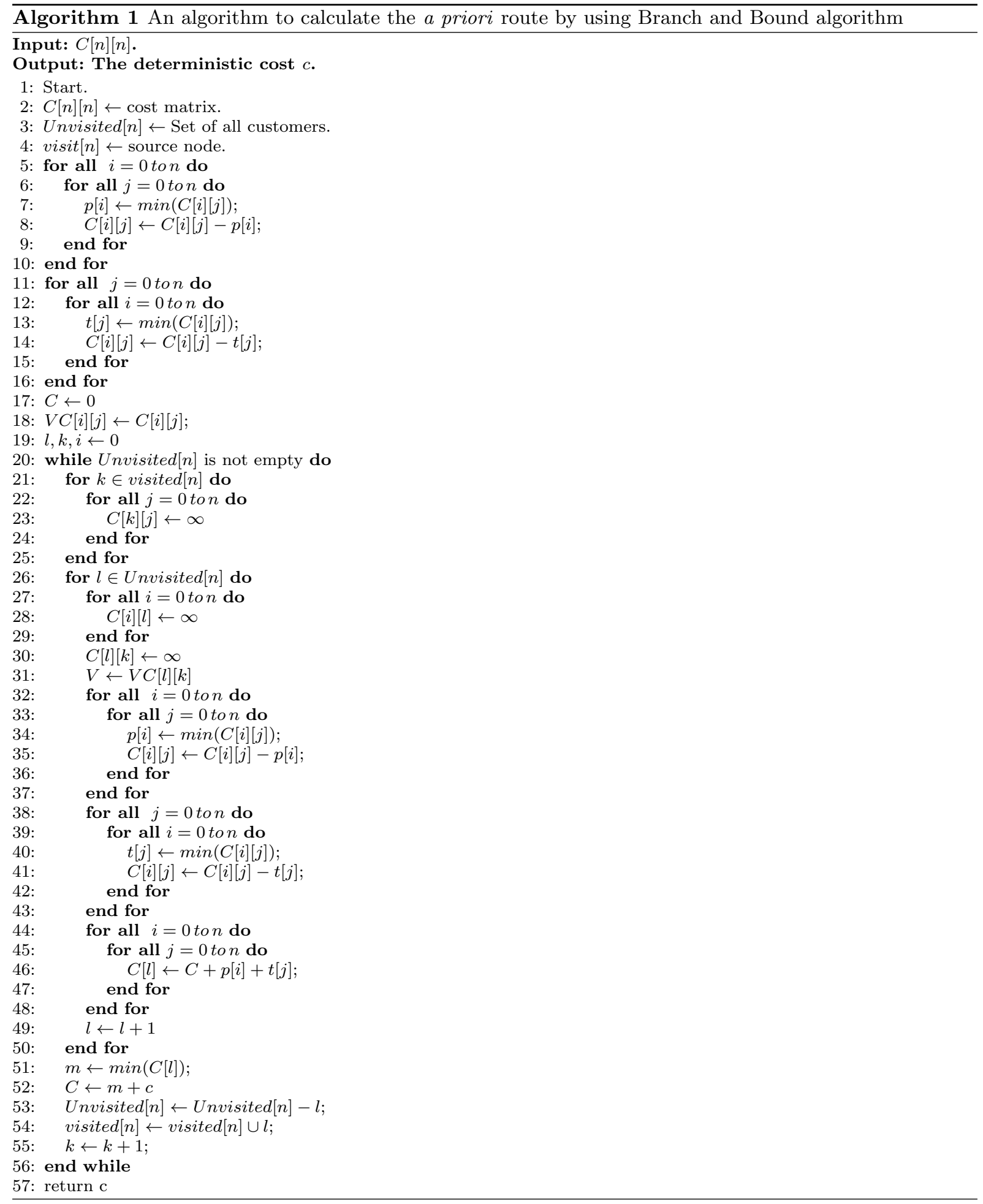



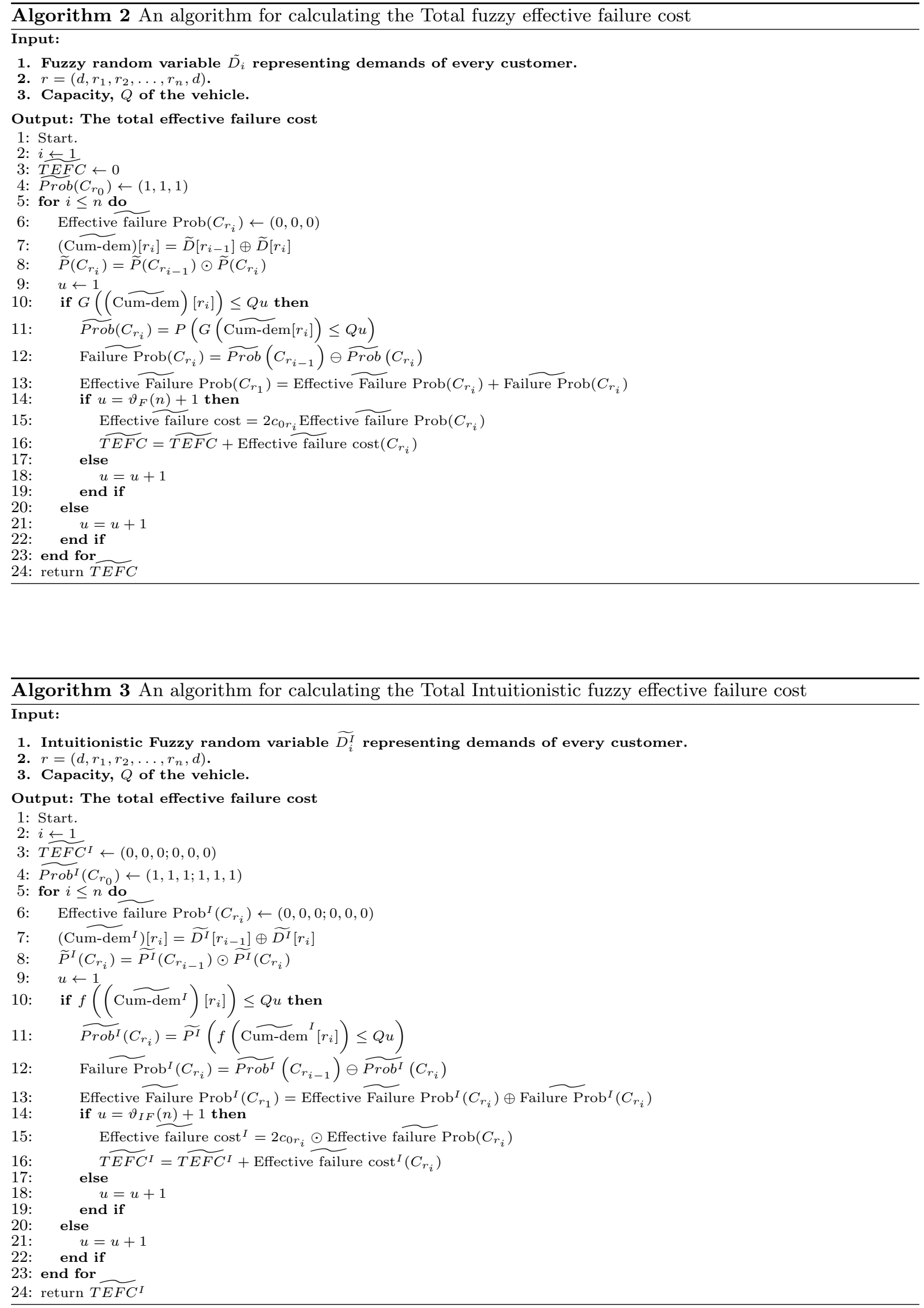


\subsection{Flowchart}

Figure 4 and Figure 5 represents the flowchart for methodology of stage 1 and stage 2 respectively.

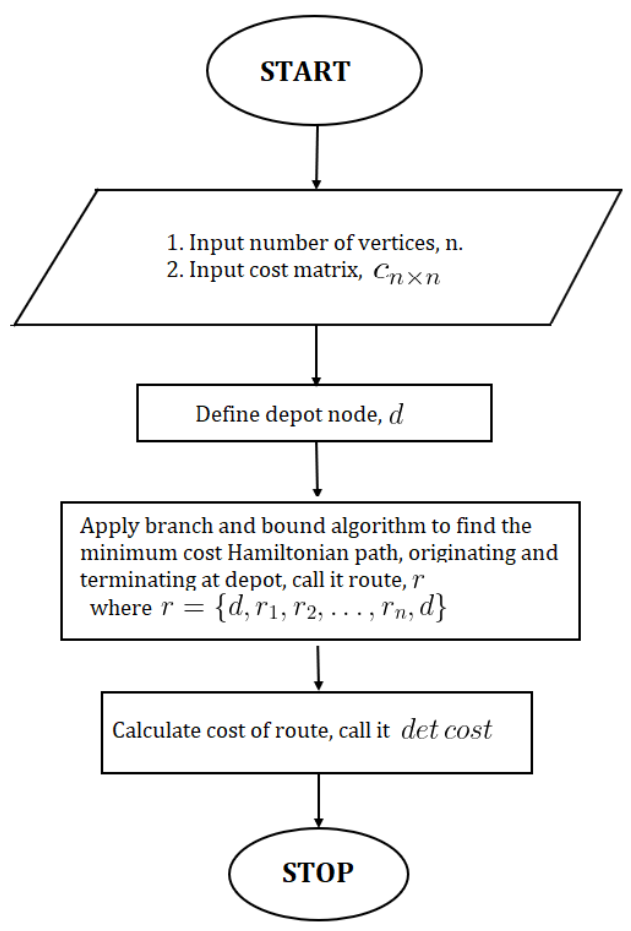

Fig. 4: Flowchart for Stage 1

\section{Result and Discussion}

\subsection{Numerical Example for CVRPFSD}

To illustrate the working of the mathematical model that has been explained in subsection 3.1, let us assume a network with 7 customers waiting for the goods to be delivered. The depot node is denoted by Node-0 and the customers are waiting at the remaining nodes. The information about the cost of traversal between various nodes is given by $C$, where

$$
C=\left[\begin{array}{cccccccc}
\infty & 4 & 8 & 3 & 9 & 6 & 5 & 11 \\
4 & \infty & 9 & 6 & 10 & 3 & 4 & 6 \\
8 & 9 & \infty & 8 & 4 & 8 & 12 & 5 \\
3 & 6 & 8 & \infty & 9 & 8 & 7 & 3 \\
9 & 10 & 4 & 9 & \infty & 11 & 12 & 2 \\
6 & 3 & 8 & 8 & 11 & \infty & 8 & 15 \\
5 & 4 & 12 & 7 & 12 & 8 & \infty & 12 \\
11 & 6 & 5 & 3 & 2 & 15 & 12 & \infty
\end{array}\right]
$$

The demand at the depot node is considered to be 0 units and the fuzzy stochastic demands for the customers in the network are presented by Table 2 . Let the carrying capacity of the vehicle is assumed to be 30 units.

In order to find the number of vehicles required, we calculate the expected fuzzy demands for the customers present in the network. The total actual cumulative demand of the network will be the sum of all expected fuzzy demands, i.e.

$$
\text { Total cumulative fuzzy demand, } \begin{aligned}
\widetilde{T D} & =\sum_{i=1}^{7} E\left[\widetilde{D_{i}}\right] \\
& =(48.51,70.91,93.30)
\end{aligned}
$$




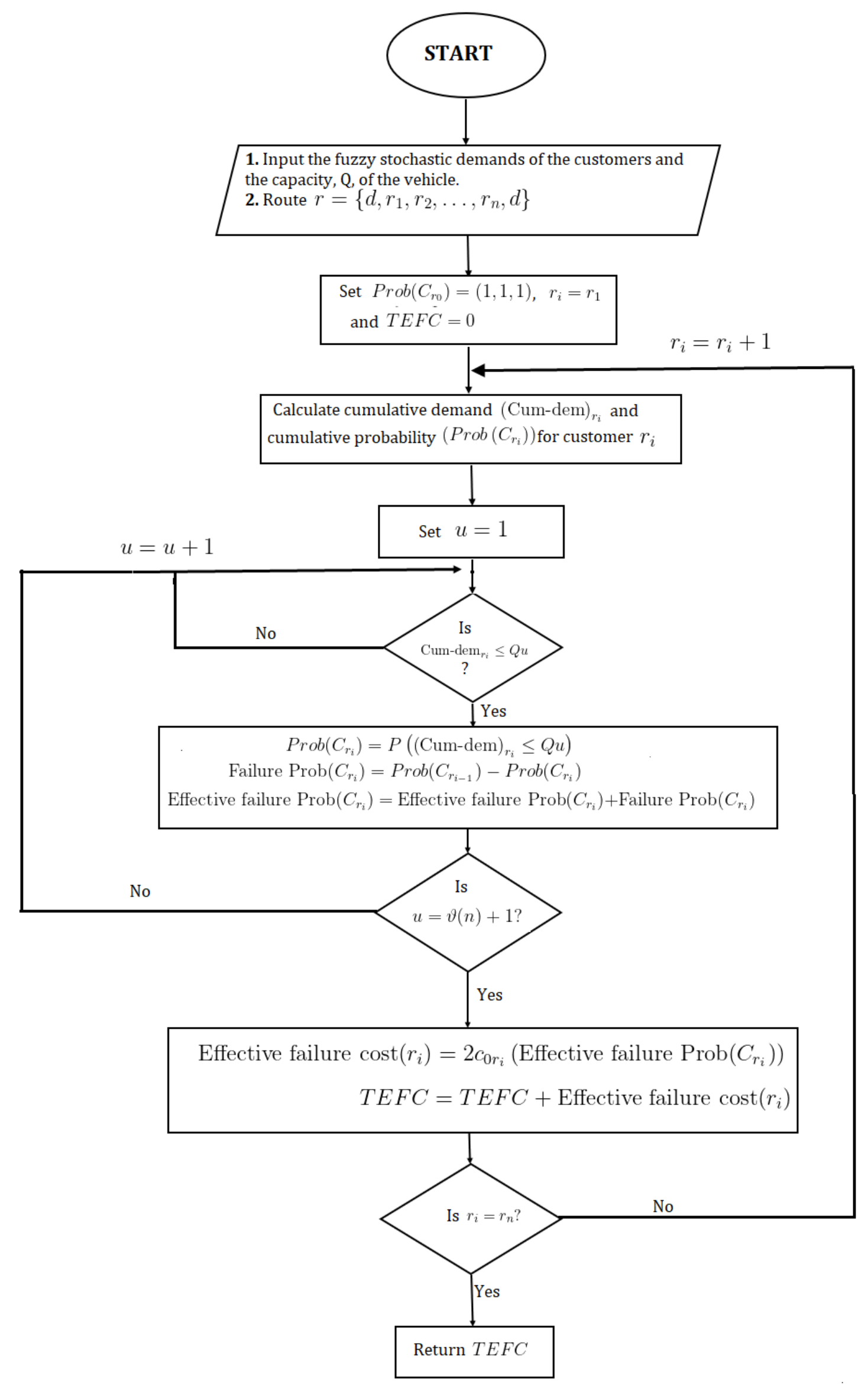

Fig. 5: Flowchart for Stage 2 
Table 2: Fuzzy Demands of Customers for CVRPFSD

\begin{tabular}{|c|c|c|}
\hline Node & Fuzzy Demands & Fuzzy Probability \\
\hline \multirow{2}{*}{1} & $\begin{array}{c}(4,6,8) \\
(8,10,12)\end{array}$ & $\begin{array}{c}(0.45,0.50,0.55) \\
(0.45,0.50,0.55)\end{array}$ \\
\hline \multirow{2}{*}{2} & $\begin{array}{c}(14,16,18) \\
(18,20,22)\end{array}$ & $\begin{array}{c}(0.75,0.80,0.85) \\
(0.15,0.20,0.25)\end{array}$ \\
\hline \multirow{2}{*}{3} & $\begin{array}{c}(8,10,12) \\
(13,15,17)\end{array}$ & $\begin{array}{c}(0.30,0.35,0.40) \\
(0.60,0.65,0.70)\end{array}$ \\
\hline \multirow{2}{*}{4} & $(8,10,12)$ & $(0.25,0.30,0.35)$ \\
& $(2,4,6)$ & $(0.65,0.70,0.75)$ \\
\hline \multirow{2}{*}{5} & $\begin{array}{c}(12,14,16) \\
(5,7,9)\end{array}$ & $\begin{array}{c}(0.35,0.40,0.45) \\
(0.55,0.60,0.65)\end{array}$ \\
\hline \multirow{2}{*}{6} & $(3,5,7)$ & $(0.58,0.63,0.68)$ \\
& $(11,13,15)$ & $(0.22,0.27,0.32)$ \\
\hline \multirow{2}{*}{7} & $(12,15,18)$ & $(0.40,0.45,0.50)$ \\
& $(4,7,10)$ & $(0.50,0.55,0.60)$ \\
\hline
\end{tabular}

Defuzzifying the above triangular fuzzy number by using Graded mean integration representation method gives

$$
\begin{aligned}
G\left(\sum_{i=1}^{7} E\left[\widetilde{D_{i}}\right]\right) & =G(48.51,70.91,93.30) \\
& =70.91
\end{aligned}
$$

In this example, the capacity of one vehicle is assumed to be 30 units and since there are chances of route failure, so it is better to approximate the number of times the vehicle should return to the source node and continue the service again. The number of times the vehicle should return to the source node can be obtained by dividing the total cumulative fuzzy demand by the capacity of the vehicle.

$$
\begin{aligned}
\vartheta\left(n_{F}\right) & =\left\lceil\frac{G\left(\sum_{i=1}^{7} E\left[\widetilde{D_{i}}\right]\right)}{Q}\right\rceil \\
& =\left\lceil\frac{70.91}{3}\right\rceil \\
& =3
\end{aligned}
$$

Thus, in order to accomplish the demands of the customers present in the network, minimum 3 vehicles are required or a single vehicle is required to make three trips. In this model, the routes are designed at $a$ priori with the help of Branch and Bound algorithm. Applying Branch and Bound algorithm on the cost matrix given by $C$, the path that the salesman should follow is given by $0 \rightarrow 6 \rightarrow 1 \rightarrow 5 \rightarrow 2 \rightarrow 4 \rightarrow 7 \rightarrow$ $3 \rightarrow 0$ and the cost incurred on travelling this route comes out to be 32 units. Till now, the route which a travelling salesperson should take if he doesn't have any demands to fulfil has been figured out. But in the model explained above, there are two constraints in stage 2, one being the maximum carrying capacity of vehicle to be 30 units and second being the fuzzy and stochastic nature of the customers' demands. , which can only be known while visiting a customer. In order to satisfy these two constraints, we find the effective failure cost at every node by using the formula given in eq. (7). We note that effective failure cost at the depot node is 0 . Table 3 comprises of comparison of method presented in this work with various other methods used for finding a priori route. The comparison has been done on the network given in the numerical example presented above.

Table 3: Comparison of Various Methods for CVRPFSD

\begin{tabular}{|l|c|c|c|c|}
\hline Name of the Method & Path & Path Cost & Effective Failure cost & Total cost \\
\hline \multirow{2}{*}{ Brute Force approach } & $0-6-1-5-2-4-7-3-0$ & 32 & $(-103.92,26.09,156.11)$ & $(-71.92,58.09,188.11)$ \\
& $0-3-7-4-2-5-1-6-0$ & 32 & $(-91.879,27.929,147.738)$ & $(-91.879,27.929,147.738)$ \\
\hline Clark and Wright Algorithm & $0-4-7-2-5-1-6-3-0$ & 41 & $(-83.52,22.688,128.90)$ & $(-42.52,63.68,169.90)$ \\
\hline Christofides Algorithm & $0-3-7-4-2-5-1-6-0$ & 32 & $(-91.879,27.929,147.738)$ & $(-59.879,59.929,179.738)$ \\
\hline Genetic Algorithm & $0-3-7-2-5-1-4-6-0$ & 49 & $(-85.69,28.063,141.824)$ & $(36.69,77.06,190.82)$ \\
\hline \multirow{2}{*}{ Bellman Held Karp Algorithm } & $0-6-1-5-2-4-7-3-0$ & 32 & $(-103.92,26.09,156.11)$ & $(-71.92,58.09,188.11)$ \\
& $0-3-7-4-2-5-1-6-0$ & 32 & $(-91.879,27.929,147.738)$ & $(-91.879,27.929,147.738)$ \\
\hline Nearest Neighbour & $0-3-7-4-2-5-1-6-0$ & 32 & $(-91.879,27.929,147.738)$ & $(-59.878,59.929,179.738)$ \\
\hline Proposed method & $0-6-1-5-2-4-7-3-0$ & 32 & $(-103.92,26.09,156.11)$ & $(-71.92,58.09,188.11)$ \\
\hline
\end{tabular}




\subsection{Numerical example for CVRPIFSD}

To illustrate the working of the mathematical model that has been explained in subsection 3.2, let us assume a network with 5 customers waiting for the goods to be delivered. The depot node is denoted by Node- 0 and the customers are waiting at the remaining nodes. The information about the cost of traversal between various nodes is given by $C_{2}$, where

$$
C_{2}=\left[\begin{array}{cccccc}
\infty & 6 & 10 & 11 & 3 & 9 \\
6 & \infty & 7 & 5 & 6 & 8 \\
10 & 7 & \infty & 9 & 8 & 7 \\
11 & 5 & 9 & \infty & 6 & 5 \\
3 & 6 & 8 & 6 & \infty & 8 \\
9 & 8 & 7 & 5 & 8 & \infty
\end{array}\right]
$$

The demand at the depot node is considered to be 0 units and the fuzzy stochastic demands for the customers in the network are presented by Table 4 . Let the carrying capacity of the vehicle is assumed to be 30 units.

Table 4: Intuitionistic Fuzzy Demands for CVRPIFSD

\begin{tabular}{|c|c|c|}
\hline Node & Intuitionistic Fuzzy Demands & Intuitionistic Fuzzy Probability \\
\hline \multirow{2}{*}{1} & $(7,8,9 ; 6,8,10)$ & $(0.25,0.30,0.35 ; 0.20,0.30,0.40)$ \\
& $(9,10,11 ; 8,10,12)$ & $(0.65,0.70,0.75 ; 0.60,0.70,0.80)$ \\
\hline \multirow{2}{*}{2} & $(10,12,14 ; 8,12,16)$ & $(0.35,0.40,0.45 ; 0.30,0.40,0.50)$ \\
& $(12,14,16 ; 10,14,18)$ & $(0.55,0.60,0.65 ; 0.50,0.60,0.70)$ \\
\hline \multirow{2}{*}{3} & $(6,7,8 ; 5,7,9)$ & $(0.40,0.45,0.50 ; 0.35,0.45,0.55)$ \\
& $(10,12,14 ; 8,12,16)$ & $(0.50,0.55,0.60 ; 0.45,0.55,0.65)$ \\
\hline \multirow{2}{*}{4} & $(13,15,17 ; 11,15,19)$ & $(0.45,0.50,0.55 ; 0.40,0.50,0.60)$ \\
& $(18,20,22 ; 16,20,24)$ & $(0.45,0.50,0.55,0.40,0.50,0.60)$ \\
\hline \multirow{2}{*}{5} & $(8,10,12 ; 6,10,14)$ & $(0.75,0.80,0.85 ; 0.70,0.80,0.90)$ \\
& $(13,15,17 ; 11,15,19)$ & $(0.15,0.20,0.25 ; 0.10,0.20,0.30)$ \\
\hline
\end{tabular}

Table 5 comprises of the comparison of the solutions obtained by various conventional methods defined in the literature for solving a TSP.

Table 5: Comparison of Various Methods for CVRPIFSD

\begin{tabular}{|c|c|c|c|c|}
\hline Name of the Method & Path & Path Cost & Effective Failure cost & Total cost \\
\hline Brute Force approach & $\begin{array}{l}0-1-3-5-2-4-0 \\
0-4-2-5-3-1-0\end{array}$ & $\begin{array}{l}32 \\
32\end{array}$ & $\begin{array}{c}(-68.49,22.40,108.28 ; \\
166.84,22.40,191.37) \\
(-63.1817,26.072,109.91 ; \\
-161.07,26.07,191.30)\end{array}$ & $\begin{array}{c}(-36.49,54.40,140.28 ; \\
-134.84,54.40,223.37) \\
(-31.1817,58.0728,141.9108 ; \\
-129.07,58.07,223.30)\end{array}$ \\
\hline Clark and Wright Algorithm & $0-1-2-3-5-4-0$ & 38 & $\begin{array}{c}(-64.47,24.32,108.07 \\
-162.04,24.32,189.64)\end{array}$ & $\begin{array}{c}(-26.47,62.32,146.07 \\
-124.04,62.32,227.64)\end{array}$ \\
\hline Christofides Algorithm & $0-5-2-3-1-4-0$ & 34 & $\begin{array}{c}(-55.70,23.93,99.23 \\
-141.55,23.93,171.96)\end{array}$ & $\begin{array}{r}(-21.70,57.93,133.23 \\
-107.55,57.93,205.96)\end{array}$ \\
\hline Genetic Algorithm & $0-4-2-1-3-5-0$ & 37 & $\begin{array}{c}(-49.57,24.37,94.04 \\
-129.63,24.37,161.16)\end{array}$ & $\begin{array}{l}(-12.57,61.37,131.04 \\
-92.63,61.37,198.16)\end{array}$ \\
\hline Bellman Held Karp Algorithm & $\begin{array}{l}0-1-3-5-2-4-0 \\
0-4-2-5-3-1-0\end{array}$ & $\begin{array}{l}32 \\
32\end{array}$ & $\begin{array}{c}(-68.49,22.40,108.28 ; \\
166.84,22.40,191.37) \\
(-63.1817,26.072,109.91 ; \\
-161.07,26.07,191.30)\end{array}$ & $\begin{array}{c}(-36.49,54.40,140.28 ; \\
-134.84,54.40,223.37) \\
(-31.1817,58.0728,141.9108 ; \\
-129.07,58.07,223.30)\end{array}$ \\
\hline Nearest Neighbour & $0-4-3-1-2-5-0$ & 37 & $\begin{array}{c}-68.185,25.404,113.79 \\
-170.914,25.404,200.59)\end{array}$ & $\begin{array}{r}(-31.185,62.404,150.79 \\
-133.914,62.404,237.59)\end{array}$ \\
\hline Proposed method & $0-1-3-5-2-4-0$ & 32 & $\begin{array}{l}(-68.49,22.40,108.28 \\
166.84,22.40,191.37)\end{array}$ & $\begin{array}{r}(-36.49,54.40,140.28 \\
-134.84,54.40,223.37)\end{array}$ \\
\hline
\end{tabular}

\subsection{Benchmark Instances}

The benchmark instances for CVRP given by Augerat in 1995(7 instances) and Christofides and Eilon in 1969 (3 instances) are modified for the imprecise and random environment. The cost of traversal of 
edges in the network is taken as the distance, which is calculated by using euclidean distance norm. The coordinates of the vertices are taken as the same given in the benchmark instances. The carrying capacity of the vehicle are considered same as that of benchmark instances. Two realizations for the fuzzy demand of the customers in the network are taken, where one realization is obtained by fuzzifying the demand as given in the benchmark instance and another realization is generated randomly in neighbourhood of first demand. The probability distribution function for customers' demand is also generated randomly. Table 6 comprises of the results of some benchmark instances obtained by using the proposed methodology.

Table 6: Results for the modified Benchmark Instances

\begin{tabular}{|c|c|c|c|}
\hline Instance & Deterministic cost & Effective failure cost & Total cost \\
\hline P-n16-k8 & 154.4 & $(-5394.58,56.83,5508.26)$ & $(-5240.189,211.236,5662.662)$ \\
\hline P-n19-k2 & 171.98 & $(-2839.51,66.39,2972.31)$ & $(-2667.53,238.37,3144.29)$ \\
\hline P-n20-k2 & 178.64 & $(-3773.39,44.56,3866.01)$ & $(-3594.75,223.20,4044.65)$ \\
\hline P-n21-k2 & 180.83 & $(-4236.45,43.19,4322.84)$ & $(-4055.62,224.02,4503.67)$ \\
\hline P-n22-k2 & 184.94 & $(-4764.50,43.056,4850.62)$ & $(-4579.56,227.99,5035.56)$ \\
\hline P-n22-k8 & 278.43 & $(-8708.64,414.93,9538.51)$ & $(-8430.21,693.36,9816.94)$ \\
\hline P-n23-k8 & 185.34 & $(-10607.27,317.25,11241.77)$ & $(-10421.93,502.59,11427.114)$ \\
\hline E-n13-k4 & 142 & $(-3178.58,114.25,3407.09)$ & $(3036.58,256.254,3549.092)$ \\
\hline E-n22-k4 & 278.43 & $(-13090.354,192.11,5474.57)$ & $(-4811.92,470.54,5753.00)$ \\
\hline E-n23-k3 & 470.04 & & $(-12569.77,659.49,13888.753)$ \\
\hline
\end{tabular}

Table 3 and Table 5 represents the comparison of various methods such as Nearest neighbour algorithm, Clark and Wright algorithm, genetic algorithm, Christofides algorithm and many more for finding the $a$ priori route and then the effective failure cost for these routes are also calculated. The sum of the effective failure cost and the deterministic cost of the routes gives the cost of operation. It has been observed that other than Branch and Bound algorithm, only Bellman Held Karp algorithm and Brute force approach gives optimal solution. The time complexity of Bellman Held Karp algorithm is exponential in nature, which is same as that of Branch and Bound algorithm. In case of Bellman Held Karp algorithm, all possible permutations are explored in all cases and hence more space in memory is required as compared to Branch and Bound algorithm. The Brute force approach gives optimal solution by exploring all possible permutations and thus have a time complexity of $\mathrm{O}(\mathrm{n} !)$, which is worse than that of Branch and Bound. Branch and Bound algorithm has time complexity of $\mathrm{O}\left(n^{2} 2^{n}\right)$, which is also exponential in nature, but no algorithm with lesser time complexity than Branch and Bound gives optimal solution for TSP. The major advantage of the algorithm is that we can control the quality of the solution to be expected, even if it is not yet found. Only in worst case scenario, the exploration of all possible permutations is required. Other algorithms such as Christofides algorithm, Nearest Neighbour algorithm, Clark and Wright algorithm, Genetic algorithm have polynomial time complexity but the solution obtained by these method is not always guaranteed to be optimal. It can be clearly observed from both the tables that the cost of operation obtained by using the proposed method is significantly lesser than the cost of operation obtained by using any other method.

\section{Conclusion}

In this work, two mathematical models for CVRPFSD and CVRPIFSD have been presented. A two stage stochastic process for solving such problems has been presented. The first stage of the problem solving corresponds to an a priori route construction. In this work, Branch and Bound algorithm has been used for this purpose. The demands of the customers are dealt in the second stage where the rote obtained in first stage is traversed. The demands of the customers present in the network are random and imprecise in nature and hence are given by discrete fuzzy random variables. An effective failure cost corresponding to every customer is calculated in the second stage. In this work, each customer is traversed exactly once and the vehicle returns to depot only when a route failure occurs, i.e. when the vehicle reaches at a customer and realizes that the customer can not be satisfied any more. The work can be used by logistic management companies to schedule the delivery of the seasonal/perishable items. Since the demands of seasonal/perishable items are stochastic in nature and various uncontrollable factors causes the impreciseness of customers' demands. This work shows new dimension of estimating the route cost in a mixed environment when information about the customer demands is based on the past experiences and includes factor of impreciseness and hesitation as well. A model for calculating the operation cost when a logical customer also includes the factor 
of hesitation while representing his/her demand is also provided in this work. Thus, the methods presented in this work deal with the modelling of randomness, impreciseness and hesitance; which a logical decision maker often encounters in real world problems. The work can further be extended for service provider companies whose objective is to provide services to the customers present at various nodes according to their specified time windows. The work can also be further extended to include randomness and impreciseness of arc lengths, such case will be helpful when the cost matrix represent the time taken to cover various edges in the network.

\section{Declarations}

Funding This research is supported by VNIT Nagpur.

Conflicts of interest/Competing interests The authors state that there is no conflict of interest.

Availability of data and material The data-sets generated during and/or analyzed during the current study are available at http://vrp.atd-lab.inf.puc-rio.br/index.php/en/.

Code availability Codes are available from the corresponding author on reasonable request.

Human and animal rights This article does not contain any studies with human participants or animals performed by any of the authors.

Informed consent Informed consent was obtained from all individual participants included in the study.

\section{References}

1. Atanassov, K.: Intuitionistic fuzzy sets. International Journal Bioautomation 20(S1), S1-S6 (2016)

2. Barma, P.S., Dutta, J., Mukherjee, A., Kar, S.: A hybrid ga-bfo algorithm for the profit-maximizing capacitated vehicle routing problem under uncertain paradigm. Journal of Intelligent \& Fuzzy Systems (Preprint), 1-17 (2021)

3. Bernardo, M., Pannek, J.: Robust solution approach for the dynamic and stochastic vehicle routing problem. Journal of Advanced Transportation 2018(1), 1-11 (2018)

4. Brito, J., Martínez, F.J., Moreno, J.A., Verdegay, J.L.: Fuzzy approach for vehicle routing problems with fuzzy travel time. In: International Conference on Fuzzy Systems, pp. 1-8. IEEE (2010)

5. Chiao, K.P.: Ranking type 2 fuzzy sets by parametric embedded representation. In: 2015 International Conference on Machine Learning and Cybernetics (ICMLC), vol. 1, pp. 371-376. IEEE (2015)

6. Chiao, K.P.: Ranking interval type 2 fuzzy sets using parametric graded mean integration representation. In: 2016 International Conference on Machine Learning and Cybernetics (ICMLC), vol. 2, pp. 606-611. IEEE (2016)

7. Clarke, G., Wright, J.W.: Scheduling of vehicles from a central depot to a number of delivery points. Operations research 12(4), 568-581 (1964)

8. Cordeau, J.F., Laporte, G., Savelsbergh, M.W., Vigo, D.: Vehicle routing. Handbooks in operations research and management science 14, 367-428 (2007)

9. Cormen, T.H., Leiserson, C.E., Rivest, R.L., Stein, C.: Introduction to algorithms. MIT press (2009)

10. Dantzig, G.B., Ramser, J.H.: The truck dispatching problem. Management science 6(1), 80-91 (1959)

11. Dorigo, M., Birattari, M., Stutzle, T.: Ant colony optimization. IEEE computational intelligence magazine 1(4), 28-39 (2006)

12. Dror, M., Laporte, G., Trudeau, P.: Vehicle routing with stochastic demands: Properties and solution frameworks. Transportation science 23(3), 166-176 (1989)

13. Dutta, J., Barma, P.S., Mukherjee, A., Kar, S., De, T., Pamučar, D., Šukevičius, Š., Garbinčius, G.: Multi-objective green mixed vehicle routing problem under rough environment. Transport pp. 1-13 (2021)

14. Fang, L., Chen, P., Liu, S.: Particle swarm optimization with simulated annealing for tsp. In: Proceedings of the 6th WSEAS International Conference on Artificial Intelligence, Knowledge Engineering and Data Bases, pp. $206-210$. Citeseer (2007)

15. Frieze, A.M.: An extension of christofides heuristic to the k-person travelling salesman problem. Discrete Applied Mathematics 6(1), 79-83 (1983)

16. Gauvin, C., Desaulniers, G., Gendreau, M.: A branch-cut-and-price algorithm for the vehicle routing problem with stochastic demands. Computers \& Operations Research 50, 141-153 (2014)

17. Gendreau, M., Laporte, G., Séguin, R.: Stochastic vehicle routing. European Journal of Operational Research 88(1), 3-12 (1996)

18. Gultom, P., Napitupulu, N.: The development of algorithm for determining optimal route for distribution of goods based on distance, time, and road quality using fuzzy set and clarke and algorithm wright savings. In: Journal of Physics: Conference Series, vol. 1542, pp. 1-9. IOP Publishing (2020)

19. Gupta, P., Govindan, K., Mehlawat, M.K., Khaitan, A.: Multiobjective capacitated green vehicle routing problem with fuzzy time-distances and demands split into bags. International Journal of Production Research pp. 1-17 (2021)

20. Irvanizam, I., Usman, T., Iqbal, M., Iskandar, T., Marzuki, M.: An extended fuzzy todim approach for multiple-attribute decision-making with dual-connection numbers. Advances in Fuzzy Systems 2020(1), 1-10 (2020) 
21. Kandasamy, V., Ilanthenral, K., Smarandache, F.: Neutrosophic graphs: a new dimension to graph theory. Infinite Study (2015)

22. Kaur, H., Singh, H.: Local search based algorithm for cvrp with stochastic demands. International Journal of Advanced Research in Computer Science 8(7), 1087-1092 (2017)

23. Kondratenko, Y., Kondratenko, G., Sidenko, I., Taranov, M.: Fuzzy and evolutionary algorithms for transport logistics under uncertainty. In: International Conference on Intelligent and Fuzzy Systems, pp. 1456-1463. Springer (2020)

24. Kuo, R., Zulvia, F.E., Suryadi, K.: Hybrid particle swarm optimization with genetic algorithm for solving capacitated vehicle routing problem with fuzzy demand-a case study on garbage collection system. Applied Mathematics and Computation 219(5), 2574-2588 (2012)

25. Laporte, G., Nobert, Y.: Exact algorithms for the vehicle routing problem. In: North-Holland Mathematics Studies, vol. 132, pp. 147-184. Elsevier (1987)

26. Marković, D., Petrovć, G., Ćojbašić, Ž., Stanković, A.: The vehicle routing problem with stochastic demands in an urban area-a case study. Facta Universitatis, Series: Mechanical Engineering 18(1), 107-120 (2020)

27. Mirzaei-khafri, S., Bashiri, M., Soltani, R., et al.: A robust optimization model for a location-arc routing problem with demand uncertainty. International Journal of Industrial Engineering 27(2), 288-307 (2020)

28. Mohammed, M.A., Ahmad, M.S., Mostafa, S.A.: Using genetic algorithm in implementing capacitated vehicle routing problem. In: 2012 International conference on computer \& information science (ICCIS), vol. 1, pp. 257-262. IEEE (2012)

29. Oyola, J.: The capacitated vehicle routing problem with soft time windows and stochastic travel times. Revista Facultad de Ingeniería 28(50), 19-33 (2019)

30. Pop, P.C., Zelina, I., Lupşe, V., Sitar, C.P., Chira, C.: Heuristic algorithms for solving the generalized vehicle routing problem. International Journal of Computers Communications \& Control 6(1), 158-165 (2011)

31. Puri, M.L., Ralescu, D.A., Zadeh, L.: Fuzzy random variables. In: Readings in Fuzzy Sets for Intelligent Systems, pp. 265-271. Elsevier (1993)

32. Russell, S.J., Norvig, P.: Artificial intelligence: a modern approach. Malaysia; Pearson Education Limited, (2016)

33. Shalaby, M.A.W., Mohammed, A.R., Kassem, S.: Modified fuzzy c-means clustering approach to solve the capacitated vehicle routing problem. In: 2020 21st International Arab Conference on Information Technology (ACIT), pp. 1-7. IEEE (2020)

34. Singh, S.K., Yadav, S.P.: Intuitionistic fuzzy multi-objective linear programming problem with various membership functions. Annals of Operations Research 269(1-2), 693-707 (2018)

35. Singh, V., Sharma, K.: Capacitated vehicle routing problem with interval type-2 fuzzy demands. In: Advances in Mechanical Engineering, pp. 83-89. Springer (2020)

36. Toth, P., Vigo, D.: The vehicle routing problem. SIAM (2002)

37. Úbeda, S., Faulin, J., Serrano, A., Arcelus, F.J.: Solving the green capacitated vehicle routing problem using a tabu search algorithm. Lecture Notes in Management Science 6(1), 141-149 (2014)

38. Werners, B., Drawe, M.: Capacitated vehicle routing problem with fuzzy demand. In: Fuzzy Sets Based Heuristics for Optimization, pp. 317-335. Springer (2003)

39. Xia, X., Liao, W., Zhang, Y., Peng, X.: A discrete spider monkey optimization for the vehicle routing problem with stochastic demands. Applied Soft Computing 111(1), 1-13 (2021)

40. Zimmermann, H.J.: Fuzzy set theory - and its applications. Springer Science \& Business Media (2011)

41. Zulvia, F.E., Kuo, R., Hu, T.L.: Solving cvrp with time window, fuzzy travel time and demand via a hybrid ant colony optimization and genetic algortihm. In: 2012 IEEE Congress on Evolutionary Computation, pp. 1-8. IEEE (2012) 